\title{
Assistenz und Wissensvermittlung am Beispiel von Montage- und Instandhaltungstätigkeiten
}

\section{Carsten Ullrich, Axel Hauser-Ditz, Niklas Kreggenfeld, Christopher Prinz und Christoph Igel}

\subsection{Einleitung}

Ein Effekt der Transformation zur Industrie 4.0 ist ein stetiger Anstieg der Komplexität sowohl in der Bedienung und Instandhaltung von Anlagen als auch in der Steuerung der Produktionsabläufe. Der sukzessive Rückgang von Produktionsmitarbeitern bei gleichzeitiger Zunahme der Komplexität der Arbeitsprozesse lässt den Informationsbedarf sowie die notwendige berufliche Expertise rasant wachsen. Intelligent-adaptive Assistenz- und Wissensdienste bieten hier Abhilfe durch die Vermittlung von formellem und informellem Wissen und Know-how, angepasst auf Expertiseniveaus und fachliche Aufgaben der Mitarbeiter.

\section{Ullrich $(\bowtie) \cdot$ C. Igel}

Educational Technology Lab (EdTec) im Deutschen Forschungszentrum für Künstliche Intelligenz (DFKI GmbH), Alt-Moabit 91c, 10559 Berlin, Deutschland e-mail: carsten.ullrich@dfki.de; christoph.igel@dfki.de

A. Hauser-Ditz

Abteilung Mitbestimmungsförderung

Hans-Böckler-Stiftung

Hans-Böckler-Str. 39, 40476, Düsseldorf

e-mail: Axel-Hauser-Ditz@boeckler.de

N. Kreggenfeld · C. Prinz

Lehrstuhl für Produktionssysteme I Fakultät für Maschinenbau

Ruhr-Universität Bochum

Universitätsstraße 150, 44801, Bochum

e-mail: kreggenfeld@lps.ruhr-uni-bochum.de; prinz@lps.ruhr-uni-bochum.de 
Das Ziel des Verbundprojektes APPsist ist die Entwicklung einer neuen Generation mobiler, kontextsensitiver und intelligent-adaptiver Assistenzsysteme zur Wissens- und Handlungsunterstützung für die Industrie 4.0. Die KI-basierten Wissens- und Assistenzsysteme unterstützen die Mitarbeiter beim Wissens- und Kompetenzerwerb in der Interaktion mit Maschinen auf dem Shopfloor.

Dieser Beitrag beschreibt die Zielsetzung des APPsist-Projektes hinsichtlich Assistenz und Lernen am Arbeitsplatz. Die sich daraus ergebende technische Lösung wird skizziert (Abschn. 8.2) und die resultierenden Veränderungen im Arbeitsprozess beispielhaft an zwei Anwendungsfällen (Montage und Instandhaltung, Abschn. 8.3) erläutert. Ein weiterer Schwerpunkt von APPsist liegt in der Konzeption von Prozessen, die die Mitarbeiter systematisch in die Entwicklung und Implementierung des Systems einbinden. Diese werden in Abschn. 8.4 beschrieben.

\subsection{Zielsetzung und technische Lösung}

Die Beherrschung der Komplexität und Flexibilität der Industrie 4.0 wird neue Formen der Arbeits- und Lernorganisation erfordern (Hirsch-Kreinsen 2014; Hirsch-Kreinsen et al. 2014). APPsist hat eine Erweiterung der Handlungsspielräume von Mitarbeitern und eine Aufwertung ihrer Arbeit zum Ziel. Konkret ruft ein Mitarbeiter das APPsist-System auf einem mobilen Endgerät auf, lässt das System individuell ausgewählte Inhalte und Tätigkeiten vorschlagen und erhält dadurch Unterstützung beim Wissenserwerb und der Durchführung bisher nicht oder wenig beherrschter Tätigkeiten. Dies ermöglicht einen Erfahrungsgewinn und das Schließen von Wissenslücken.

Ausgehend von dieser Zielsetzung wurde eine technische Plattform entwickelt, die anpassbar ist an die spezifischen Anforderungen des jeweiligen Einsatzortes, also das Unternehmen oder die Werkhalle (Ullrich 2015). Die APPsist-Plattform setzt sich aus einer Reihe von weitgehend unabhängigen, dedizierten Diensten zusammen, welche ereignisbasiert und asynchron agieren: Auslöser für die Verarbeitung und Bereitstellung von Informationen sind Ereignisse, welche entweder von Nutzern (z. B. Benutzereingaben), dem Kontext (z. B. Änderungen des Maschinenstatus) oder den Diensten selbst (z. B. basierend auf eingetragenen Regeln) kommen. Zu den zentralen Diensten der APPsist-Plattform gehören

- der Maschinen-Informationsdienst, der die Schnittstelle zwischen der Plattform und den Maschinen auf dem Shopfloor aufbaut;

- der Maßnahmendienst, der Nutzern basierend auf Maschinen- und Nutzerkontexten durchzuführende Maßnahmen vorschlägt;

- der Inhalte-Interaktionsdienst, der die Funktionalitäten der verschiedenen Dienste über verschiedene Endgeräte zugänglich macht;

- der Performance-Support-Dienst, welcher die Nutzer durch den Assistenzprozess führt;

- die Prozess-Koordinationsinstanz, welche Assistenzprozesse ausführt und verwaltet und

- der Inhalte-Selektor, welcher die relevanten Lern-und Assistenz-Inhalte auswählt. 
Abb. 8.1 Benutzeroberfläche APPsist

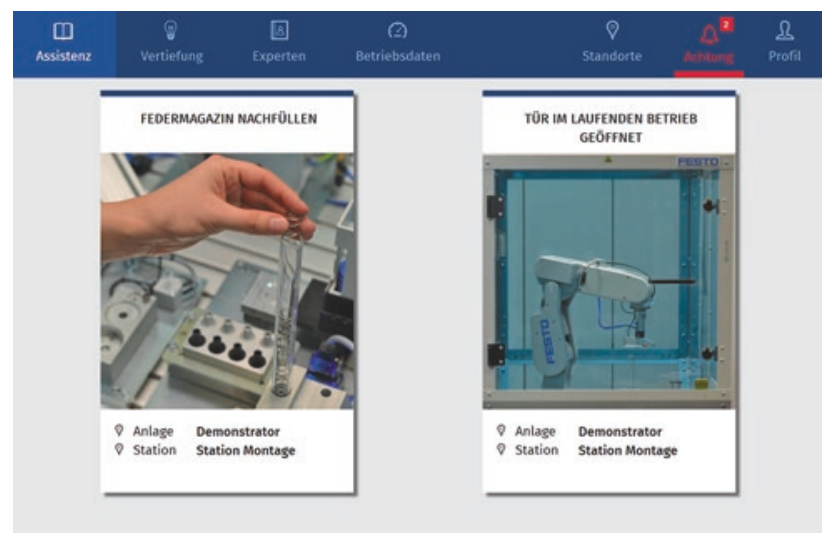

Abb. 8.1 zeigt die Oberfläche des APPsist-Systems. Der Benutzer befindet sich im Menüpunkt „Assistenz“ und bekommt daher eine Auswahl von aktuell durchführbaren Maßnahmen angezeigt.

Die Zielsetzung einer intelligent-adaptiven Unterstützung, die also angepasst an den individuellen Nutzer und an die spezifische Maschinenkonfiguration und -situation eine geeignete Unterstützung vorschlägt, erfordert dementsprechend ,intelligente“ Dienste. Systeme, die sich mithilfe von Methoden der Künstlichen Intelligenz flexibel an individuelle Lernende anpassen, können als konkrete Instanzen eines allgemeinen Entwurfsmusters aufgefasst werden, das auf drei Komponenten basiert: einem Wissensmodell, einem Lernermodell und einem Bildungsmodell. Das Wissensmodell besteht aus dem in Lernobjekte strukturierten und mit Metadaten annotierten Lehr-Lern-Material. Das Lernermodell enthält eine Abschätzung des Wissensstandes, der Eigenschaften etc. der Lernenden, die als Basis der Personalisierung und individuellen Fortschrittskontrolle dient. Um Passung zwischen Wissensmodell und Lernermodell zu erzeugen, verfügen diese Systeme weiterhin über ein Bildungsmodul, in dem didaktisches Wissen in einer computerverwendbaren Form (z. B. als Expertensystem) formalisiert ist.

In APPsist wurde die Domäne „Produktion“ modelliert, d. h. die auftretenden Konzepte und deren Zusammenhänge wurden in einer formalen Beschreibungssprache beschrieben und in einer semantischen Datenbank abgelegt. Abb. 8.2 zeigt beispielhaft einen vereinfachten Ausschnitt des Domänenmodells, der die für die Assistenz- und Wissensdienste relevanten Produktionsgegenstände beschreibt.

Dieses Modell dient als eindeutiges und festgelegtes Vokabular der Kommunikation der Dienste und als Grundlage für die intelligenten Entscheidungsprozesse der adaptiven Dienste. So kann beispielsweise der Maßnahmendienst über generische Anfragen an die semantische Datenbank die für den aktuellen Kontext relevanten Maßnahmen bestimmen.

Das Lernermodell enthält Informationen darüber, wie oft ein Benutzer mit einem Konzept des Domänenmodells interagiert hat, d. h. wie oft ein Assistenzschritt gestartet, abgebrochen und beendet wurde, wie oft ein Lerninhalt oder Dokument angesehen wurde, und 


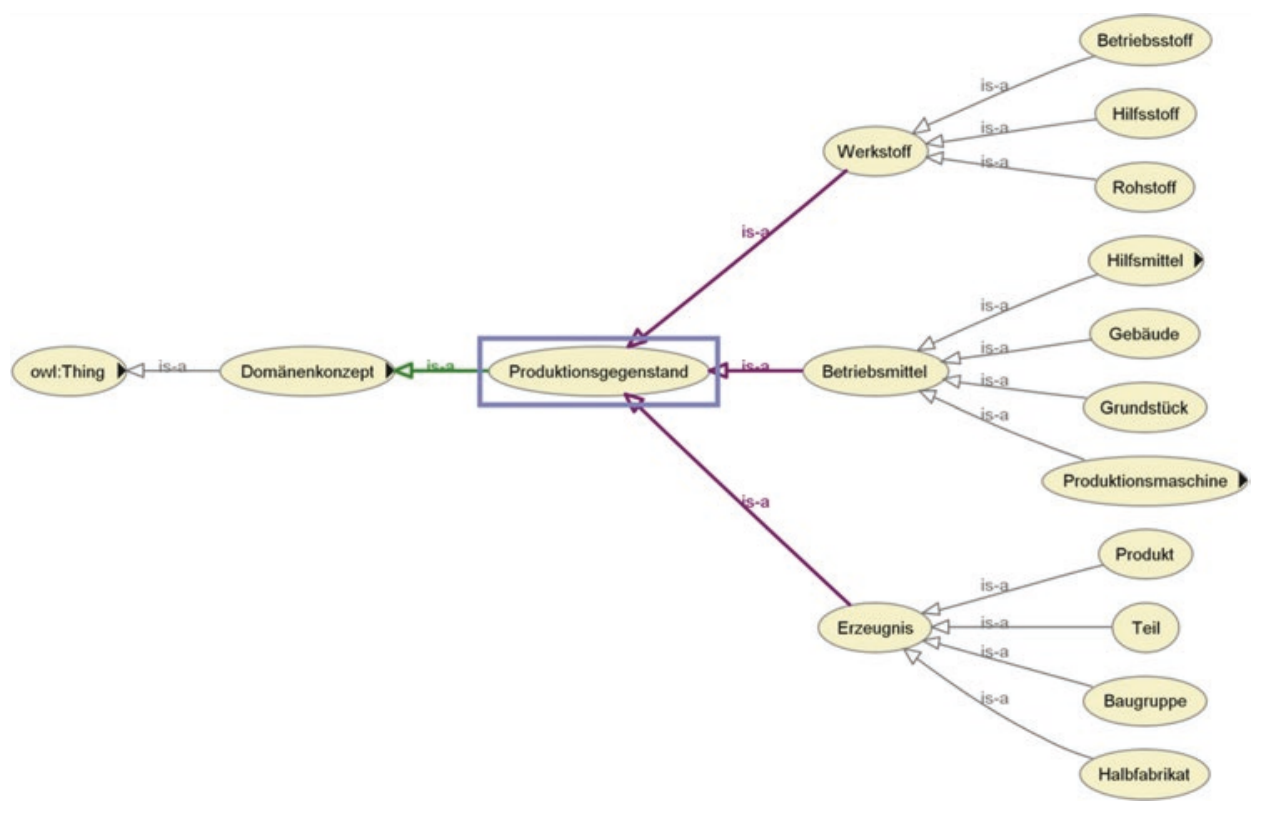

Abb. 8.2 Domänenmodell APPsist (Auszug)

wie oft ein Produktionsgegenstand verwendet wurde. Zusätzlich werden weitere relevante Benutzerinformation gespeichert, nämlich die Arbeitsplatzgruppe des Mitarbeiters, Freigaben (welche Tätigkeiten der Mitarbeiter durchführen darf), ob er/sie sich aktuell in der Haupttätigkeit (beispielsweise der Montage) oder Nebentätigkeit „Lernzeit“ befindet (zu Lernzeiten siehe auch Abschn. 8.4) und welches die Entwicklungsziele sind. Das Modell geht davon aus, dass Entwicklungsziele in Zielvereinbarungsgesprächen zwischen Mitarbeiter und Führungskraft festgelegt werden. Entwicklungsziele können konkrete Inhalte und/oder eine neue Beschäftigungsgruppe und/oder Produktionsgegenstände beinhalten.

Das Bildungsmodell realisiert die Adaptivität bezogen auf die Dimensionen ,Zeithorizont“ und „Art der Unterstützung“. Der Zeithorizont umfasst die beiden Werte ,,aktueller Zustand“ (der Mitarbeiter soll bei einer durch den momentan geltenden Zustand bedingten Arbeit unterstützt werden) und „Entwicklungsperspektive“ (der Mitarbeiter wird beim Erreichen eines langfristigen Zieles unterstützt). Der erste Fall ist beispielsweise eine Reaktion auf konkret auftretende Probleme im Betrieb: Meldet eine Maschine, dass ein benötigter Werkstoff (z. B. Loctite) nicht mehr in ausreichendem Maße vorhanden ist, so wird der Mitarbeiter darauf hingewiesen und bekommt passende Maßnahmen im APPsist-System angezeigt. Im zweiten Fall geschieht die Unterstützung bezogen auf die im Mitarbeitergespräch vereinbarten Entwicklungsziele. So kann das APPsist-System Inhalte und Maßnahmen bezogen auf langfristige Entwicklungsperspektiven vorschlagen. Die Art der Unterstützung kann in einer Assistenz bestehen, d. h. bei der konkreten Durchführung einer Tätigkeit wie dem Wechseln von Werkstoffen, oder in der Bereitstellung von 
Inhalten. Es lassen sich also im aktuellen APPsist-System folgende vier Adaptionsregeln unterscheiden (in der tatsächlichen technischen Implementierung setzen sich diese Regeln aus mehreren Teilregeln zusammen):

- Haupttätigkeit-Assistenz (HA): Wenn Mitarbeiter in „Haupttätigkeit“ und fordert Assistenz an, dann bestimme Maßnahmen, die relevant sind für aktuelle Station und Maschinenzustand.

- Nebentätigkeit-Assistenz (NA): Wenn Mitarbeiter in „Nebentätigkeit Lernzeit“ und fordert Assistenz an, dann bestimme Maßnahmen, die relevant sind für langfristige Entwicklungsperspektive.

- Haupttätigkeit-Inhalte (HI): Wenn Mitarbeiter in „Haupttätigkeit“ und Anforderung von Inhalten, dann bestimme Inhalte, die relevant sind für aktuelle Station und Maschinenzustand.

- Nebentätigkeit-Inhalte (NI): Wenn Mitarbeiter in „Nebentätigkeit Lernzeit“ und Anforderung von Inhalten, dann bestimme Inhalte, die relevant sind für langfristige Entwicklungsperspektive.

\subsection{Betriebliche Anwendungsfälle}

\subsubsection{Hintergrund/Motivation}

$\mathrm{Zu}$ Demonstrations- und Validierungszwecken wurden im APPsist Forschungsprojekt in Zusammenarbeit mit industriellen Anwendungspartnern verschiedene Anwendungsszenarien entwickelt. Diese wurden bewusst so definiert, dass sie hinsichtlich der relevanten Parameter „Zielgruppe“ und „Komplexität“ deutlich variieren, um so die Leistungsfähigkeit und Breitenwirksamkeit des APPsist-Systems zu verdeutlichen.

Zur Darstellung der erweiterten Befähigung zur Ausführung von zusätzlichen Tätigkeiten (z. B. einfache Instandhaltung) wurde ein Anwendungsszenario ausgewählt, bei dem der Wechsel eines Werkstoffes in einer teilautomatisierten Montagelinie nicht mehr durch eine Fachkraft (z. B. Mechatroniker), sondern durch eine dort tätige angelernte Montagekraft (Zielgruppe) mithilfe von Assistenz (geringe Komplexität) durchgeführt werden soll. Die Zielsetzung dabei ist, an- oder ungelernte Mitarbeiter/Innen dazu zu befähigen, diesen Prozess selbstständig und effizient durchzuführen. Durch diese Umverteilung von Aufgaben und Verantwortlichkeiten sollen Anlagenstillstände reduziert und somit Kosten eingespart werden. Angelernte Kräfte können mittels der Assistenz flexibler eingesetzt werden und Fachkräfte können zu einem höheren Zeitanteil Aufgaben mit höheren Anforderungen bearbeiten.

Zur Betrachtung der komplexeren Assistenz zur Reduzierung der Bearbeitungszeit und Qualitätserhöhung/Fehlervermeidung wurde ein Anwendungsszenario im Vorrichtungsbau gewählt, bei dem komplexe Montagetätigkeiten im Vorrichtungsbau durch Fachkräfte (z. B. Industriemechaniker, Elektrotechniker) mittels Assistenzsystemen unterstützt 
werden. Im Gegensatz zum ersten Anwendungsszenario bilden hier hochqualifizierte Mitarbeiter die Zielgruppe. Die Komplexität dieses Prozesses liegt in der hohen Individualität der zu montierenden Vorrichtungen (mechanische, pneumatische und elektronische Komponenten). Diese sind modular aufgebaut, unterscheiden sich jedoch im Detail je nach Kundenanforderung signifikant, sodass eine kundenbezogene Beschreibung der korrelierenden Montagetätigkeiten erfolgen muss. Letztendlich sollen so Verschwendungen sowie Fehler im Prozess und somit auch die benötigte Montagezeit reduziert und die Qualität erhöht werden.

Für die Bereitstellung von Assistenz ist es zunächst erforderlich, die jeweiligen Prozesse eindeutig und nachvollziehbar zu beschreiben. Die Prozessaufnahme erfolgt in workshoporientierten Meetings, an denen im Wesentlichen die Experten für den zu definierenden Prozess teilnehmen. Diese können je nach Prozess und Unternehmensgröße durch Fachkräfte, Führungskräfte und Geschäftsführer repräsentiert werden. Als Prozessbeschreibungssprache kommt die Business Process Model and Notation (BPMN) zum Einsatz (BPMN 2011). Definiert werden die jeweiligen relevanten Prozessschritte in unterschiedlichen, zielgruppenorientierten Detaillierungsstufen.

Die zu beschreibenden Prozesse werden zunächst in einer Maßnahme (oberste Ebene) definiert. Die Maßnahme setzt sich aus verschiedenen Aktivitäten zusammen, welche somit die zweite Ebene darstellen. Diese lassen sich wiederum weiter in eine Folge von Aktionen (dritte Ebene) untergliedern. Die dritte Ebene setzt sich aus elementaren Schritten zusammen. Diese maximal dreistufige Detaillierung gewährleistet zum einen die Übersichtlichkeit der Prozessstruktur, zum anderen ermöglicht sie aber auch, Mitarbeitern im Rahmen der Assistenzgebung Informationen in unterschiedlicher Granularität und somit Assistenz für unterschiedliche Fähigkeitsstufen bereitstellen zu können. Um die Prozesse schließlich für die Verwendung im Assistenzsystem aufzubereiten, werden den einzelnen Prozessschritten auf allen Ebenen Assistenzinhalte zugeordnet. Diese bestehen im Wesentlichen aus einem Text, der dem Mitarbeiter Informationen darüber liefert, was im entsprechenden Schritt getan werden muss. Hinzu kommen je nach Prozessschritt auch notwendige Warnsymbole, Hinweise sowie unterstützende Fotos, Videos, Modelle oder technische Zeichnungen. Je nach Anwendungsfall sind hier auch AR-Inhalte (Augmented Reality) möglich.

\subsubsection{Beschreibung der assistierten Prozesse und der Unterstützung}

Nachfolgend wird das Anwendungsbeispiel „Werkstoff wechseln“ beschrieben. Dieser Prozess ist in Abb. 8.3 auf allen drei Detaillierungsebenen (Maßnahme, Aktivität, Aktion) grafisch dargestellt. Auf der obersten Ebene ist der Prozess bewusst sehr übersichtlich gestaltet. Hier ist lediglich beschrieben, dass die Station angehalten und nach dem Wechsel wieder in Betrieb genommen werden muss. Außerdem müssen die Reste des entnommenen Stoffes ordnungsgemäß entsorgt werden. Um den Prozess für die entsprechende Zielgruppe adäquat zu beschreiben, ist daher eine Detaillierung auf der Aktivitäten- und Aktionsebene unabdingbar. Hier wird Schritt für Schritt darauf eingegangen, welche Einzelaktivitäten zur Durchführung des Prozesses notwendig sind. Entsprechend 


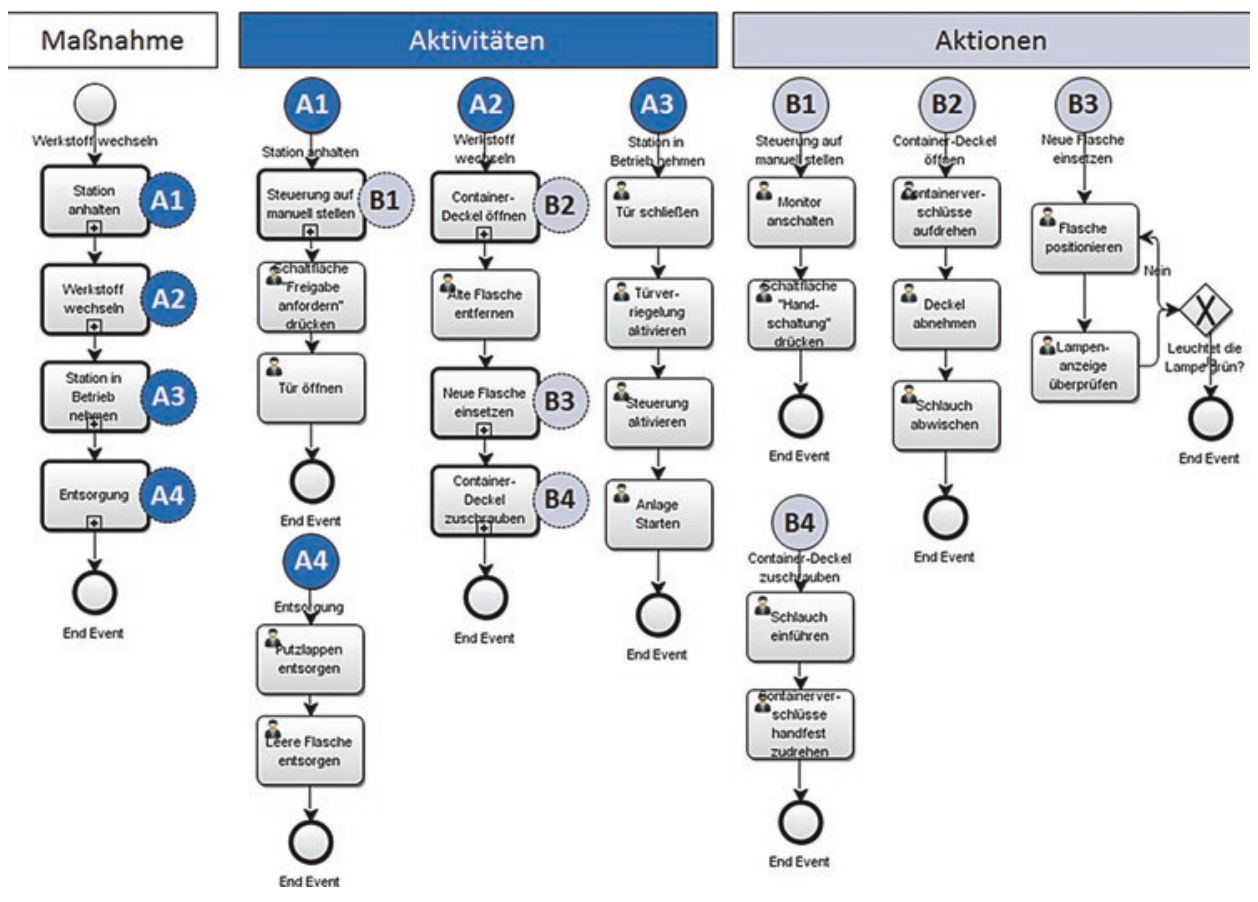

Abb. 8.3 Prozessdarstellung in BPMN

aufbereitete Fotos und Videos werden dem Mitarbeiter angezeigt. Gerade bei dem eigentlichen Wechsel- und Entsorgungsvorgang sind darüber hinaus aber auch Warnhinweise notwendig, die auf die Gefahren im Umgang mit dem Stoff sowie auf Vorschriften hinsichtlich der zu treffenden Vorsichtsmaßnahmen aufmerksam machen.

Die Komplexität beim zweiten Anwendungsszenario, dem Montageprozess im Vorrichtungsbau, der von einer Fachkraft durchgeführt wird, liegt weniger in der Beschreibung der einzelnen Prozessschritte, da diese im Wesentlichen bei der angesprochenen Zielgruppe bekannt sind. Vielmehr geht es hier um die korrekte Zuordnung der anzuzeigenden Inhalte, die in diesem Fall hauptsächlich aus technischen Zeichnungen bestehen. Hier kann keine starre Zuordnung zwischen Inhalten und Prozessschritten erfolgen, sondern diese muss sich stets am Kunden und der korrelierenden Variante der Vorrichtung orientieren. Somit ist eine direkte Vernetzung des Assistenzsystems mit den Automatisierungssystemen (z. B. ERP, MES) notwendig.

Um die verschiedenen Arten der Unterstützung, die durch das APPsist-System möglich sind, zu veranschaulichen, wird im Folgenden davon ausgegangen, dass sich der angelernte Montagemitarbeiter in der Nebentätigkeit "Lernzeit“ und die Fachkraft in der Haupttätigkeit befindet.

Fordert der Montagemitarbeiter Frank Müller Assistenz an, werden die im Lernermodell abgelegten Entwicklungsziele herangezogen: Die Führungskraft hat bemerkt, dass Herr Müller über erhebliches technisches Geschick verfügt. So hat Herr Müller in den 
letzten Wochen, unterstützt durch die APPsist-Assistenz, erfolgreich verschiedene Werkstoffe gewechselt. Es wurde daher vereinbart, dass der Mitarbeiter sich strategisch auf die Übernahme weiterer Instandhaltungsaufgaben vorbereiten soll. Ihm werden also vom APPsist-System weitere, etwas komplexere Maßnahmen zur Instandhaltung wie beispielsweise „Problem bei Schraubenzufuhr beheben“ vorgeschlagen (Regel NA). Wählt er eine Maßnahme aus, kann er diese, geleitet von der Assistenz, beispielsweise an einer Lerninsel oder in einer Lernfabrik vorab simulieren.

Wählt hingegen die Fachkraft Sabine Meyer Unterstützung durch Assistenz in der Haupttätigkeit aus (Regel HA), werden ihr auf den aktuellen Kontext bezogene Maßnahmen vorgeschlagen, in diesem Fall beispielsweise ,pneumatische Komponenten montieren“. Fordert Frau Meyer Inhalte an (Regel HI), dann werden für die momentane Situation

relevante Dokumente ausgewählt, z. B. der pneumatische Schaltplan für den spezifischen Kunden oder Lerninhalte zu pneumatischer Montage.

Im Falle von Herrn Müller werden bei Inhaltsanforderung (Regel NI) vereinbarte Inhalte (Informationen über das Unternehmen, das Produkt und den Produktionsprozess) und hinsichtlich der Entwicklungsziele relevante Inhalte (Beschreibung der Aufgaben eines Instandhalters etc.) angezeigt.

\subsubsection{Veränderungen im Arbeitsprozess durch die Einführung des Assistenzsystems}

In den derzeitigen Pilotszenarien lassen sich bereits einige Tendenzen für die Veränderung von Tätigkeiten und der Organisation von Arbeit ausmachen (Tab. 8.1). Diese entsprechen den Zielvorstellungen innerhalb des Projektes. Damit diese Effekte sich auch in einem großflächigen Einsatz des Systems bestätigen, bedarf es der fortlaufenden Beobachtung, Anpassung und Optimierung und darauf basierender Gestaltung.

Bei der Darstellung der Veränderungen wird zunächst der oben beschriebene Anwendungsfall der Assistenz für einfache Instandhaltungstätigkeiten (Wechsel eines Werkstoffs) in einer teilautomatisierten Montagelinie (Spalte 1) fokussiert.

Personalbedarf/Übertragung von Tätigkeiten zwischen Arbeitsplätzen und betrieblichen Funktionen: Im konkreten Anwendungsfall werden einzelne Tätigkeiten der vorbeugenden Instandhaltung vom Anlagenführer (Facharbeit) auf die Anlagenbediener (angelernte Montagemitarbeiter) übertragen. Diese werden mittels Assistenz in die Lage versetzt, ausgewählte komplexere Tätigkeiten auszuführen. Hierbei handelt es sich weiterhin um standardisierbare Routinetätigkeiten. Ihre Ausführung umfasst jedoch im Unterschied zur kurz getakteten Routinetätigkeit am Montagearbeitsplatz mehr Arbeitsschritte und diese fallen vergleichsweise selten, bspw. im Abstand von mehreren Tagen an.

Durch die Übertragung von Tätigkeiten an die Montagemitarbeiter sinkt für diese insgesamt der Anteil monotoner (Montage)Arbeiten. Gleichzeitig nimmt der Komplexitätsgrad der Tätigkeit zu und es werden mehr problemlösende und kontrollierende Tätigkeiten 
Tab. 8.1 Auswirkungen auf Tätigkeiten und Kompetenzanforderungen

\begin{tabular}{l|l|l}
\hline Veränderungen & $\begin{array}{l}\text { Szenario1: einfache } \\
\text { Teilmontage durch angelernte } \\
\text { Montage-MA }\end{array}$ & $\begin{array}{l}\text { Szenario 2: komplexe } \\
\text { Werkzeugmontagedurch } \\
\text { Fachkraft }\end{array}$ \\
\hline Personalbedarf & 0 & 0 \\
\hline Tätigkeitsveränderungen & ++ & 0 \\
\hline Problemlösung & - & 0 \\
\hline Monotone Aufgaben & + & 0 \\
\hline Komplexe Aufgaben & 0 & 0 \\
\hline Planen & + & - \\
\hline Kontrolltätigkeiten & ++ & + \\
\hline Lernen, informell & + & + \\
\hline Lernen, formell & + & 0 \\
\hline Selbstbestimmung & + & + \\
\hline Optimierung & + & + \\
\hline Kooperation & & + \\
\hline Kompetenzanforderungen & + & + \\
\hline Kommunikation & + & 0 \\
\hline Interdisziplinarität & 0 & + \\
\hline IT-Kenntnisse & & + \\
\hline
\end{tabular}

-- wird viel weniger, - wird weniger, 0 bleibt gleich, + wird mehr, ++ wird viel mehr.

übernommen. Dies bedeutet jedoch nicht automatisch, dass die Erweiterung von Tätigkeiten auch mit einem erhöhten Maß an Selbstbestimmung am Arbeitsplatz einhergeht, da die erweiterten Aufgaben und Problemlösungen jeweils mehr oder weniger strikt durch das System vorgegeben werden. Hier kommt es auf die konkrete Ausgestaltung des APPsist-Systems an. Zu erwarten ist zudem, dass auch Prozesse der Selbstoptimierung der Montagemitarbeiter durch das System unterstützt werden. Auch dürfte die Kooperation zwischen den Einzelarbeitsplätzen in der Montagelinie und zwischen den unterschiedlichen Funktionsbereichen zunehmen, da durch den Einsatz des mobilen Systems mehr Abstimmungsprozesse anfallen (wer die konkrete Maßnahme ausführen soll, Wissensaustausch über von Kollegen/Innen hinterlegte Erfahrungsberichte, Kontaktaufnahme mit Technikern bei Störungen etc.).

Mit der Übertragung von Tätigkeiten auf die Montagemitarbeiter werden auch Effekte für andere Mitarbeitergruppen entstehen. Es wird insgesamt eine aufwertende Verschiebung im Tätigkeitsgefüge ausgelöst (Fahrstuhleffekt). So soll etwa der Maschinenführer künftig einzelne Aufgaben übernehmen, die bisher bspw. den Instandhaltern vorbehalten waren - etwa die Beseitigung von vergleichsweise häufig auftretenden mechanischen 
Störungen. Die Instandhalter wiederum erhalten zusätzliche Aufgaben in einem zunehmend technisierten Umfeld (perspektivisch steigender Automatisierungsgrad). Letztlich fallen auch zusätzliche Arbeiten bei der Pflege des Assistenzsystems an. Zum einen entsteht zusätzlicher Arbeitsaufwand für die Generierung neuer Unterstützungsszenarien, die zunächst maschinenlesbar in BPMN modelliert werden müssen. Zum anderen ist zu erwarten, dass bei breitflächiger Implementierung des Systems auch ein erhöhter Instandhaltungsbedarf für das System selbst entsteht. Insgesamt werden durch den Einsatz des Assistenzsystems die Tätigkeiten an den Montagearbeitsplätzen wie auch auf der Ebene des Anlagenführers und des Wartungspersonals tendenziell anspruchsvoller. Eindeutige Beschäftigungseffekte für die unterschiedlichen betrieblichen Funktionen lassen sich derzeit noch nicht prognostizieren.

\section{Qualifizierungsbedarf und Qualifizierungsmöglichkeiten:}

Das Assistenzsystem ist für den Benutzer selbsterklärend und wird in dieser Hinsicht umfangreich mit ausgewählten Mitarbeitern des Pilotbereichs getestet und optimiert. Ein unmittelbarer Qualifizierungsbedarf für die Handhabung des Systems ergibt sich auf dieser Ebene zunächst nicht. Dessen ungeachtet ist allein schon aus arbeitsschutzrechtlichen Anforderungen eine Unterweisung in die Systemhandhabung und die assistierten Prozesse nötig. Hierzu gehört auch, die Mitarbeiter über die Rahmenbedingungen des Systemeinsatzes (bspw. Regelungen zum Datenschutz, zur Leistungskontrolle etc.) sowie über den grundsätzlichen Aufbau der verwandten Modelle (Transparenz darüber, welche Daten wozu verwendet werden und welche Folgen ein bestimmtes Nutzerverhalten hat) zu informieren.

Im Unterschied zu anderen, meist stationären Assistenzsystemen im Montagebereich, beinhaltet das APPsist-System weitere Dienste, die über die unmittelbare Anleitung von Tätigkeiten hinausgehen. Insbesondere eröffnen sich durch die Integration von Lernmaterial und weiteren Informationsressourcen neue Möglichkeiten für arbeitsplatznahes Lernen (siehe oben).

Effekte könnten sich auch für die erforderlichen kommunikativen Kompetenzen ergeben. In dem Maße, wie Kooperations- und Kommunikationsschnittstellen innerhalb des Systems realisiert werden (im aktuellen Stadium sind noch nicht alle vorgesehenen Funktionalitäten implementiert) und Abstimmungsprozesse bspw. mit Instandhaltern oder IT-Services auftreten, erhöhen sich auch die Anforderungen an kommunikative Kompetenzen.

Demgegenüber stellen sich bei den Systembenutzern zunächst keine erhöhten Qualifikationsanforderungen in Bezug auf IT-Kenntnisse - jedenfalls auf der Ebene der einfachen Montagearbeitsplätze. Höhere Qualifizierungsanforderungen könnten aber bei anderen Beschäftigtengruppen entstehen, etwa bei den Systeminstandhaltern. Hier wird es davon abhängen, bei welchen Arbeitsplätzen die Pflege des Assistenzsystems angesiedelt ist bzw. wie hier gegebenenfalls auch einzelne Aufgaben (Aufnahme neuer Prozesse, Beheben von Störungen im Assistenzsystem, Weiterentwicklung der Systemarchitektur) verteilt werden. 
Höhere Anforderungen an Kommunikation und soziale Kompetenz könnten sich zudem noch auf einer anderen Ebene ergeben: Sofern Entwicklungsziele von Mitarbeitern tatsächlich über Qualifizierungsgespräche und Zielvereinbarungen festgelegt werden sollen, müssen auch die Vorgesetzten hierfür geschult sein. Auch für die Mitarbeiter selbst wäre, wie Erfahrungen aus anderen Unternehmen zeigen, eine Vorbereitung auf das Qualifizierungsgespräch sinnvoll.

Für die im Beispiel beschriebenen Fachkräfte im Vorrichtungsbau ergibt sich bezüglich der Veränderungen ihrer Tätigkeiten ein anderes Bild. Hier zeichnet sich weniger eine komplett neue Tätigkeitsstruktur mit gänzlich neuen Anforderungen, sondern vielmehr eine Optimierung und Unterstützung der bestehenden Arbeitsabläufe ab. Durch die Verfügbarkeit der richtigen Inhalte und Informationen zur richtigen Zeit am richtigen Ort können ineffiziente Abläufe reduziert werden. So müssen beispielsweise Schaltpläne nicht mehr zentral auf einem Bildschirm neben dem Montagearbeitsplatz abgerufen werden, sondern stehen direkt auf dem Tablet vor Ort bereit. Ebenso werden langwierige Vorbereitungen durch das Suchen der korrekten, also zum Kunden passenden, Pläne als Arbeitsvorbereitung reduziert sowie Inkonsistenzen durch Versionsupdates solcher Zeichnungen eliminiert. Der Mitarbeiter kann sich somit vollständig auf die eigentliche, wertschöpfende Montagetätigkeit konzentrieren.

Zusammenfassend skizziert Tab. 8.2 die prognostizierten Veränderungen: Durch die Erweiterung der Arbeitsaufgaben für Montagekräfte wird es am Layout der Arbeitsplätze nur geringfügige Änderungen geben, da es sich beim Arbeitsmittel nicht um ein stationäres, sondern um ein mobiles Endgerät handelt. Dieses wird beispielsweise innerhalb einer U-förmigen Montagelinie bzw. an einer außenliegenden Station zur Nutzung angebracht. Veränderungen müssen insofern erfolgen, als dass ein geeigneter Ort für die Bearbeitung von Lerneinheiten außerhalb der eng geführten Assistenzschritte insbesondere für die Nebentätigkeit Lernzeit gefunden werden muss. Die Arbeitsorganisation muss so angepasst werden, dass Lernzeiten planbar zur Verfügung stehen. Führungsstrukturen ändern sich nicht, wohl aber die Anforderungen an unmittelbare Vorgesetzte in Bezug auf die systematische Gestaltung von Qualifizierungsgesprächen und Zielvereinbarungen.

\subsection{Projektansatz: Beteiligungsorientierte Entwicklung und Implementierung von Assistenzlösungen}

Das Projekt APPsist verfolgt einen beteiligungsorientierten Ansatz bei der Entwicklung und Implementierung des Systems, um einerseits mögliche Akzeptanzprobleme frühzeitig zu erkennen, andererseits Potenziale zur Systemoptimierung aus dem späteren Benutzerumfeld während der Entwicklungs- und Testphase einspeisen zu können. Der Beteiligungsansatz bezieht sich sowohl auf die späteren Systembenutzer als auch auf die betrieblichen Arbeitnehmervertreter, die im Prozess der Systemeinführung und -ausgestaltung aufgrund der betriebsverfassungsrechtlichen Vorgaben vielfältige Informations- und Mitbestimmungsrechte haben. 
Tab. 8.2 Zusammenfassende Betrachtung der Auswirkungen

\begin{tabular}{|c|c|}
\hline Arbeitsaufgabe & $\begin{array}{l}\text { Neue (höherwertige) Aufgaben kommen hinzu und müssen } \\
\text { unterstützt werden }\end{array}$ \\
\hline Arbeitstätigkeiten & $\begin{array}{l}\text { Neue Tätigkeiten werden ermöglicht } \\
\text { Tätigkeiten werden unterstützt/erläutert } \\
\text { Seltene, repetitiv auftretende Tätigkeiten können unterstützt } \\
\text { und in Erinnerung gerufen werden }\end{array}$ \\
\hline Arbeitsplatz/Arbeitsraum & $\begin{array}{l}\text { Montagearbeitsplätze bleiben zunächst unverändert, da } \\
\text { Einsatz des Assistenzsystems an automatisierter Station } \\
\text { Veränderungen ggf. bei Einsatz als Lernsystem am } \\
\text { Arbeitsplatz }\end{array}$ \\
\hline Arbeitsmittel & $\begin{array}{l}\text { Bisherige Arbeitsmittel (Werkzeuge, Pläne etc.) werden } \\
\text { ergänzt bzw. ersetzt durch ein intelligentes, mobiles } \\
\text { Assistenzsystem (Tablet, APPs etc.) }\end{array}$ \\
\hline Arbeitsorganisation & $\begin{array}{l}\text { Muss angepasst werden: } \\
\text { Neue Tätigkeiten; Tätigkeitserweiterung } \\
\text { Zeitmodelle für Lerneinheiten } \\
\text { Zeitmodelle für Wissensaufbau }\end{array}$ \\
\hline Arbeitsumgebung & $\begin{array}{l}\text { Mittelbare Auswirkungen auf Zusammenarbeit möglich } \\
\text { (z. B. Anwender vs. Nicht-Anwender) }\end{array}$ \\
\hline Qualifikation/Kompetenz & $\begin{array}{l}\text { Kompetenzniveau wird angehoben; operativ (Assistenz) } \\
\text { sowie nachhaltig (Wissenselemente) } \\
\text { MA mit formell gleicher Qualifikationsstufe können } \\
\text { komplexere Tätigkeiten ausführen } \\
\text { System als vielseitig einsetzbares Tool für arbeitsplatznahes } \\
\text { Lernen (Inhalte erweiterbar) }\end{array}$ \\
\hline Führung & $\begin{array}{l}\text { Zunächst keine direkten Auswirkungen auf } \\
\text { Führungsstrukturen }\end{array}$ \\
\hline
\end{tabular}

\subsubsection{Beteiligung der Benutzer an der Systemgestaltung}

Zur Vorbereitung der schrittweisen Systemimplementierung, der eine längere Erprobungsund Verbesserungsphase vorausgeht, wurde in einem der Anwenderunternehmen eine Testgruppe gebildet. Diese ist intensiv in die Erprobung der unterschiedlichen Entwicklungsstadien des Assistenzsystems eingebunden. Die Gruppe besteht aus einem repräsentativen Querschnitt von Montagekräften und Maschinenführern. Sie wurde auf der Grundlage einer Rahmenvereinbarung der Betriebsparteien (siehe unten) auf freiwilliger Basis konstituiert. Das Interesse der Beschäftigten im Pilotbereich zur Mitarbeit in der Testgruppe wird von allen Projektbeteiligten als hoch eingeschätzt. 
Die Gruppe ist dabei ausdrücklich aufgefordert, eigene Vorschläge in die Systemgestaltung einzubringen. Erste Tests der Benutzeroberfläche fanden bereits statt. In der nächsten Erprobungsstufe wird mit den Beteiligten ein Test der oben dargestellten Assistenzszenarien im realen Produktionsumfeld und des im Entwicklungskonsortium erarbeiteten Lernermodells in unterschiedlichen Varianten erfolgen.

Hierbei wird durch die beteiligten Projektpartner sowie durch die Prozessbeteiligung des Betriebsrats sichergestellt, dass auftretende Fragen und Probleme wie auch Verbesserungsvorschläge in einem strukturierten Dialog bearbeitet werden können.

\subsubsection{Absicherung des betrieblichen Rahmens durch Einbindung der Arbeitnehmervertretung}

Bei der Systemeinführung sind grundlegende Beteiligungs- und Mitbestimmungsrechte des Betriebsrats berührt (u. a. $\$ 80$ und $\$ 87$ (1) BetrVG). Eine frühzeitige Beteiligung der Arbeitnehmervertreter ist daher ohnehin geboten. Diese wurde im Projekt gewährleistet, indem zwei Betriebsratsmitglieder als ständige Repräsentanten in das Projektkonsortium aufgenommen wurden, die somit einen Zugang zum Projektstand haben. Auf Initiative des Betriebsrats eines Anwenderunternehmens wurde zudem auf betrieblicher Ebene eine Rahmenvereinbarung (Regelungsabrede) abgeschlossen, in der die Bedingungen für die Entwicklungs- und Erprobungsphase des Assistenzsystems festgeschrieben wurden. Dies betraf den zeitlichen Ablauf, die Bildung einer freiwilligen Testgruppe und die Kommunikationswege während der Testphase. Im Wesentlichen ging es darum, eventuell entstehende Nachteile für Benutzer des Systems von vornherein auszuschließen und somit präventiv die Systemakzeptanz zu erhöhen. Für den Betriebsrat ging es darum, die Zielrichtung des Projektes - über die bei den Arbeitnehmervertretern hohe Unsicherheit bestand - symbolisch und für die Mitarbeiter sichtbar abzusichern. Hierbei kam es auch zu einer Einschränkung der nutzbaren Endgeräte. Der Einsatz von Datenbrillen, die aufgrund ihrer ergonomischen Risiken und den mit dieser Technologie verbundenen erleichterten Überwachungsmöglichkeiten auf Vorbehalte bei den Betriebsräten stießen, ist in der Testphase nicht vorgesehen. Vereinbart wurde zudem, aus den im Erprobungsprozess gesammelten Erfahrungen, vor der tatsächlichen Implementierung des Systems in den Regelbetrieb, gemeinsam den weiteren Regelungsbedarf festzustellen und notwendige Vereinbarungen zu treffen. In diesem Prozess der Ausarbeitung betrieblicher Vereinbarungslösungen werden die Betriebsparteien durch eine wissenschaftliche Begleitung und durch die IG Metall unterstützt, die innerhalb des Projektes mit der konzeptionellen Unterstützung der Arbeitnehmervertreter betraut ist. Letztlich soll es darum gehen, Musterlösungen zu finden, die für ähnlich gestaltete Assistenzsysteme auch in anderen Unternehmenskontexten anwendbar sind. 


\subsubsection{Betrieblicher Regulierungsbedarf im Rahmen der Systemimplementierung}

Im Rahmen der Systemimplementierung sind neben rein organisatorischen auch eine Reihe regulatorischer Fragen zu klären. Diese werden im Projektkonsortium vorgeklärt, bedürfen aber letztlich verbindlicher Absprachen und Vereinbarungen zwischen den betrieblichen Akteuren. Dabei geht es im Wesentlichen um folgende Themen:

1. Ein erster Themenkomplex betrifft unmittelbar Fragen von Ergonomie und Arbeitsschutz. Hier gilt es sicherzustellen, dass die Endgeräte und Benutzeroberflächen gesicherten arbeitswissenschaftlichen Erkenntnissen entsprechen und vor Inbetriebnahme eine Gefährdungsbeurteilung des Gesamtsystems durchgeführt wird. Hierzu müssen die Akteure des betrieblichen Arbeitsschutzes (Betriebsleitung, Betriebsrat, Fachkraft für Arbeitssicherheit, Sicherheitsbeauftragter des Bereichs) mit Unterstützung des Projektkonsortiums gemeinsam Implementierungslösungen erarbeiten.

2. Ein zweiter zentraler und für die Mitarbeiter sensibler Themenkomplex ist der Datenschutz und die mit der Generierung von mitarbeiter- und prozessbezogenen Daten entstehenden vielfältigen Möglichkeiten der Überwachung und Leistungskontrolle. Hier sind zentrale Mitbestimmungsrechte des Betriebsrats ( $\$ 87$ (1) 6 BetrVG) berührt, die eine Vereinbarung zwischen Betriebsrat und Arbeitgeber zwingend erfordern. Auch auf der Seite der Mitarbeiter, die das System nutzen sollen, kann die Frage, wozu die erhobenen Daten verwendet werden und welche Auswirkungen die Datensammlung für sie hat, Akzeptanzprobleme hervorrufen. Daher wird im Projekt schon in der Entwicklungsphase großer Wert auf Transparenz hinsichtlich des Datenmodells (welche Daten sollen erhoben und zu welchem Zweck gespeichert werden) und der Adaptitvitätsregeln (welche Folgen haben einzelne Handlungen) gelegt und nach Möglichkeiten der Entkopplung von Personen- und Prozessdaten gesucht. Dies wird eine Regelung zum Datenschutz und zur Datennutzung zwischen den Betriebsparteien nicht überflüssig machen aber wesentlich erleichtern.

3. Ein dritter Themenkomplex betrifft Qualifizierungsfragen. Hier gilt es, geeignete Regelungen für den Einsatz des Lernsystems am Arbeitsplatz zu entwickeln. Schließlich sind dabei komplexe Sachverhalte betroffen. Dies beginnt bei der Klärung organisatorischer Fragen, die sich auf die praktische Durchführbarkeit von Lernen im Arbeitsprozess beziehen (geeignete Lernorte, Lage und Anrechnung von Lernzeiten). Hierbei muss nach geeigneten Modellen gesucht werden, die auch die Restriktionen geltender tarifvertraglicher Rahmenbedingungen (TV Bildung ${ }^{1}$ ) berücksichtigen.

\footnotetext{
${ }^{1}$ Tarifvertrag Bildung in der Metall- und Elektroindustrie 2015 löst den Tarifvertrag Qualifizierung von 2006 ab.
} 
4. Schließlich ergibt sich bei dem hier vorgestellten Modell, das auf eine Aufwertung von Arbeitsplätzen setzt, auch die Frage nach der künftigen Bewertung von assistierten Tätigkeiten - zumindest dann, wenn diese den Gesamtcharakter der Tätigkeiten am betreffenden Arbeitsplatz nachhaltig verändern. Kann eine solche Aufwertung auch zur Höherbewertung der betroffenen Arbeitsplätze führen? Dies muss perspektivisch auf der Basis der bestehenden tarifvertraglichen Regelwerke (ERA ${ }^{2}$ ) zwischen den Betriebsparteien geklärt werden. Unter Umständen ergeben sich auch Entwicklungsbedarfe für das Regelwerk selbst, etwa wenn Facharbeitertätigkeiten durch den Einsatz von Assistenz teilweise von angelernten Kräften übernommen werden können. Dies soll unter Einbeziehung von Fachexperten geprüft werden.

\subsection{Zusammenfassung}

In diesem Beitrag wurden Assistenz- und Wissensdienste für Mitarbeiter in der Produktion und ihre Auswirkungen auf Arbeitsprozesse an konkreten Anwendungsbeispielen beschrieben. Ein Assistenzdienst kann zu monotonerer Arbeit führen, durch strikte Prozessvorgaben den Handlungsspielraum von Mitarbeitern einschränken oder auch abwechslungsreichere Arbeit ermöglichen und arbeitsplatznahe Lernprozesse unterstützen. Die in APPsist angelegte technische Infrastruktur aus mobilen Endgeräten, konkreter Prozessunterstützung und hiermit vernetzten Wissensbeständen eröffnet große Möglichkeiten für das zweite Szenario. Allein die technische Bereitstellung der Unterstützungsdienste hat dabei noch keine eindeutigen Auswirkungen auf die Arbeitsprozesse. Für die konkrete Ausprägung ist das Zusammenspiel zwischen technischer und organisatorischer Implementierung entscheidend. Daher werden in APPsist auch Leitfäden für die Systemausgestaltung und -implementierung sowie Bausteine für Musterbetriebsvereinbarungen entwickelt werden, die eine qualifikationsfördernde und Handlungsspielräume erweiternde betriebliche Realisierung ermöglichen.

Ein ebenfalls relevanter Punkt, der im Projektverlauf bearbeitet werden wird, ist die weitere Ausarbeitung der Unterstützung ,höherwertiger“ Tätigkeiten. Das Potenzial des APPsist-Systems für Fachkräfte liegt weniger in der Assistenz beim Durchführen von prozessbezogenen Tätigkeiten (welche Aktion ist in diesem Schritt notwendig?), als vielmehr bei der Unterstützung von Tätigkeiten, die sie als Experten charakterisieren, also bei der Wissensverknüpfung, Wissensgenerierung, Prozessoptimierung etc. Es werden also möglicherweise andere Dienste und auch andere Adaptionsregeln für diese Zielgruppe nötig werden.

\footnotetext{
${ }^{2}$ Entgeltrahmentarifvertrag der Metall- und Elektroindustrie. Dieser enthält einen umfangreichen Katalog zur Bewertung von Tätigkeiten und Aufgaben.
} 


\subsection{Förderkennzeichnung}

Das diesem Bericht zugrunde liegende Forschungs- und Entwicklungsprojekt wird mit Mitteln des Bundesministeriums für Wirtschaft und Energie (BMWi) innerhalb des Technologieprogramms „AUTONOMIK für Industrie 4.0" unter dem Förderkennzeichen 01MA13004A gefördert und vom Projektträger ,,Technische Innovationen in der Wirtschaft“" im Deutschen Zentrum für Luft- und Raumfahrt, Köln betreut. Die Verantwortung für den Inhalt dieser Veröffentlichung liegt bei den Autoren.

\section{Literatur}

BPMN. (2011). Technical Report. Business Process Model and Notation (BPMN) Version 2.0. Object Management Group.

Hirsch-Kreinsen, H. (2014). Wandel von Produktionsarbeit - Industrie 4.0. Dortmund: Technische Universität Dortmund, Wirtschafts- und Sozialwissenschaftliche Fakultät.

Hirsch-Kreinsen, H., Ittermann, P., \& Niehus, J. (Hrsg.) (2014). Digitalisierung industrieller Arbeit. Die Vision von Industrie 4.0 und ihre sozialen Voraussetzungen. Baden-Baden: Edition Sigma/Nomos.

Ullrich, C., Aust, M., Blach, R., Dietrich, M., Igel, C., Kreggenfeld, N., Kahl, D., Prinz, C., \& Schwantzer, S. (2015). Eine Architektur für intelligent-adaptive Assistenz- und Wissensdienste in der Industrie 4.0. In S. Schäfer (Hrsg.), Industrie 4.0: Grundlagen und Anwendungen; Branchentreff der Berliner Wissenschaft und Industrie (S. 111-123). Berlin [u. a.]: Beuth.

Open Access Dieses Kapitel wird unter der Creative Commons Namensnennung 4.0 International Lizenz (http://creativecommons.org/licenses/by/4.0/deed.de) veröffentlicht, welche die Nutzung, Vervielfältigung, Bearbeitung, Verbreitung und Wiedergabe in jeglichem Medium und Format erlaubt, sofern Sie den/die ursprünglichen Autor(en) und die Quelle ordnungsge-mäß nennen, einen Link zur Creative Commons Lizenz beifügen und angeben, ob Änderungen vorgenommen wurden.

Die in diesem Kapitel enthaltenen Bilder und sonstiges Drittmaterial unterliegen ebenfalls der genannten Creative Commons Lizenz, sofern sich aus der Abbildungslegende nichts anderes ergibt. Sofern das betreffende Material nicht unter der genannten Creative Commons Lizenz steht und die betreffende Handlung nicht nach gesetzlichen Vorschriften erlaubt ist, ist für die oben aufgeführten Weiterverwendungen des Materials die Einwilligung des jeweiligen Recht-einhabers einzuholen.

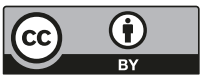

